\title{
Riqueza e diversidade de aves aquáticas de uma lagoa natural no sudeste do Brasil
}

\author{
Marcos Rodrigues \& Vania B. Michelin
}

Laboratório de Ornitologia, Departamento de Zoologia, Universidade Federal de Minas Gerais. Caixa Postal 486, 31270 901 Belo Horizonte, Minas Gerais, Brasil. E-mail: ornito@mono.icb.ufmg.br

\begin{abstract}
Species richness and diversity of waterbirds of a natural lake in southeastern Brazil. Increases and decreases of waterfowl population have been used as an indicator of water quality and habitat quality. The aim of this paper was to survey and monitor waterfowl population at the largest natural lake of the 'Area of Environmental Protection' of Lagoa Santa, Southeast Brazil. The 'Lagoa do Sumidouro' holds approximately 253 ha with a $12,072 \mathrm{~m}$ of perimeter. Species richness and abundance was estimated from June 1999 to December 2002. It was recorded 27 species of 12 families. Twelve species were considered residents, while eight migratory and another seven species did not present any seasonal pattern. Ardeidae was the most representative family holding $23 \%$ of the species followed by Anatidae (15\%) and Socolopacidae (11\%). Platalea ajaja Linnaeus, 1758 is considered threatened to extinction, while Mycteria americana Linnaeus, 1758 and Ciconia maguari (Gmelin, 1789) are considered rare for the state of Minas Gerais. There was no difference of species richness among the three main seasons ('wet', ‘dry', and 'transitional'). Species diversity was higher during the wet season. The occurrence of threatened and migratory species at 'Lagoa do Sumidouro' makes the lake an area of extreme biological importance.
\end{abstract}

KEY WORDS. Biodiversity, bioindicator, Cerrado, migration, Platalea ajaja, Minas Gerais.

RESUMO. O aumento ou decréscimo das populações de aves aquáticas tem sido usado como indicador da qualidade de água e conseqüentemente da qualidade ambiental. $O$ objetivo do presente trabalho foi o de inventariar e monitorar a avifauna aquática da maior lagoa da região do sistema cárstico de Lagoa Santa, região central de Minas Gerais, dentro da 'Área de Proteção Ambiental Carste de Lagoa Santa' (APA LS), a Lagoa do Sumidouro. A Lagoa ocupa uma área de aproximadamente $253 \mathrm{~h}$ na época de maior cheia e apresenta um perímetro de cerca de 12.072 m. Durante o período de junho de 1999 a dezembro de 2002 foram estimadas a riqueza e a abundância das aves aquáticas presentes na Lagoa. Foram registradas 27 espécies de aves aquáticas, distribuídas em 12 famílias. Das 27 espécies registradas, 12 foram consideradas residentes ou prováveis residentes e oito visitantes. Sete espécies não apresentaram nenhum padrão distinto de presença sazonal. A família mais bem representada foi Ardeidae com seis espécies perfazendo $23 \%$ do total de espécies. Em seguida, a família Anatidae com cinco espécies (15\%) e Scolopacidae sendo representada por três espécies ( $11 \%$ do total). Uma das espécies Platalea ajaja Linnaeus, 1758 é considerada ameaçada de extinção no Estado de Minas Gerais, enquanto outras duas Mycteria americana Linnaeus, 1758 e Ciconia maguari (Gmelin, 1789) são consideradas raras. Não houve diferença significativa entre a riqueza de espécies estimada para as três estações ('chuvosa', 'seca' e 'transição'). A diversidade de espécies mostrou-se significativamente diferente entre as três estações, sendo a estação chuvosa com a maior diversidade. A presença de espécies ameaçadas de extinção e local de parada de espécies migratórias faz com que a região da APA Carste de Lagoa Santa seja considerada uma área de extrema importância referente aos aspectos biológicos.

PALAVRAS CHAVE. Biodiversidade, Bioindicador, Cerrado, migração, Platalea ajaja, Minas Gerais.

O maior obstáculo para a formulação e compreensão de modelos ecológicos que estimam a viabilidade em longo prazo das Unidades de Conservação (UC) e Áreas de Proteção Ambiental (APA) nas regiões tropicais tem sido a falta de informação sobre a estrutura populacional das espécies de animais e plantas a serem conservados, ou seja, sua demografia (FernANDez 1997). Uma solução seria usar um grupo taxonômico do qual se possa extrair esse tipo de dado com facilidade e extrapolar esse conhecimento para se prever a viabilidade das UC's (Soulé 1987). Nesse contexto, as Aves podem desempenhar papel fundamental, uma vez que elas estão entre os vertebrados com maior número de informações sobre sua biologia, taxonomia e distribuição geográfica (Pough et al. 1999).

O monitoramento de populações de aves produz dados não

Revista Brasileira de Zoologia 22 (4): 928-935, dezembro 2005 
apenas para formulação de modelos de populações mínimas viáveis, mas também fornece dados sobre diminuição e aumento populacional, bem como sobre migração e qualidade do habitat. Assim, a presença ou ausência de certas espécies, bem como as tendências populacionais podem ser usadas como indicadores de qualidade ambiental (GoldsMith 1991, FuRness et al. 1993).

O aumento ou decréscimo das populações de aves aquáticas tem sido usado como indicador da qualidade de água (RUTSCHKE 1987). Isto porque estas populações são influenciadas por mínimas alterações do habitat como drenagem, aterramento, desmatamento, sobrepesca, poluição, e alterações do fluxo de água como aqueles provenientes de construções de barreiras.

O objetivo do presente trabalho foi o de fazer um levantamento da avifauna aquática que ocorre na maior lagoa da região do sistema cárstico de Lagoa Santa, região central de Minas Gerais, dentro da 'Área de Proteção Ambiental Carste de Lagoa Santa' (APA LS). Embora a APA LS seja internacionalmente reconhecida como uma das localidades mais importantes da América do Sul sob os pontos de vista botânico (WARMING 1908), arqueológico (Neves et al. 1998) e paleontológico (CARTelle 1994), pouca ênfase vem sendo dada ao sistema cárstico como um todo. A região abriga um sistema complexo de mais de 60 lagoas conectadas por canais subterrâneos naturais. Estas lagoas passam por um ciclo de seca e cheia que afeta a dinâmica de flora e fauna associadas a elas. Sendo assim, além do levantamento de espécies, nosso escopo foi o de monitorar as populações destas aves por um período de três anos para se verificar o papel das estações do ano na sua dinâmica de ocupação da lagoa.

Existem dois levantamentos de aves feitos na região da APA LS, sendo o primeiro feito no século XIX pelo naturalista J. Reinhardt, cuja lista está publicada em WARMING (1908) e PINто (1952). O segundo foi feito entre os anos de 1996 e 1997 por ocasião do zoneamento ambiental da APA (HERMAnN et al. 1998). Esses dois levantamentos e o presente trabalho deverão formar uma base de dados que servirá de subsídio para a implementação de qualquer projeto de manejo de fauna dentro da APA LS.

\section{MATERIAL E MÉTODOS}

\section{Área de estudo}

O trabalho foi desenvolvido na região da Lagoa do Sumidouro localizada entre os municípios de Pedro Leopoldo e Lagoa Santa, em Minas Gerais ( $\left(9^{\circ} 32^{\prime} \mathrm{S}\right.$ e $\left.43^{\circ} 47^{\prime} \mathrm{W}\right)$, cerca de $50 \mathrm{~km}$ ao norte de Belo Horizonte. Esta área faz parte da APA Carste Lagoa Santa, uma das áreas brasileiras mais importantes em termos de paisagem cárstica carbonática e da história das ciências naturais do país. A APA Carste Lagoa Santa apresenta grande quantidade de dolinas muitas vezes limitadas por paredões calcáreos lineares e grandes maciços rochosos aflorantes ou parcialmente encobertos (PILó 1997). Também percebe-se na região muitos lagos com diferentes comportamentos hídricos associados as dolinas ou em amplas planícies rebaixadas que podem ser alagadas sazonalmente (SouzA 1998). Além disso, a região cárstica de Lagoa Santa é uma das poucas regiões do planeta cujo siste- ma hidrológico de lagoas é formado pela conexão de canais subterrâneos naturais de uma lagoa à outra (SouzA 1998).

A Lagoa do Sumidouro é o maior ambiente lacustre da APA Carste Lagoa Santa (SouzA 1998). Ocupa uma área de aproximadamente 253 ha na época de maior cheia e apresenta um perímetro de cerca de 12.072 metros. As formações vegetais predominantes no entorno são de Cerrado e Floresta Estacional Semidecidual (Hermann et al. 1998). O cerrado restringe-se à manchas remanescentes, em regeneração ou em transição (mata-cerrado). Nas dolinas e arredores dos afloramentos prevalece a Floresta Estacional Semidecidual. Sobre os afloramentos calcários desenvolve-se Floresta Estacional Decidual ("mata seca") (Hermann et al. 1998).

Esta lagoa sofre profundas alterações no volume de água, de acordo com o índice pluviométrico (Souza 1998). Em períodos de seca, o corpo d'água diminui consideravelmente e grande parte de sua extensão é invadida por uma vegetação baixa que é utilizada como pasto pelos animais das propriedades rurais localizadas nas proximidades. Com o aumento dos índices pluviométricos na estação chuvosa, a lagoa se restabelece.

A temperatura média do ar é da ordem de $23^{\circ} \mathrm{C}$, sendo $11,2^{\circ} \mathrm{C}$ a média das mínimas temperaturas num período de 30 anos (mês de julho), e 29, $6^{\circ} \mathrm{C}$ a média das máximas (outubro a março). A umidade relativa varia de $60 \%$ a $77 \%$ nos meses mais secos e úmidos, respectivamente, chegando a 96\% nos meses mais úmidos.

As estações seca e chuvosa e as transições foram determinadas a partir do trabalho de Madeira \& Fernandes (1999). Estação seca refere-se aos meses de maio a setembro; estação chuvosa de novembro a janeiro; e estação de transição de fevereiro a abril e outubro. Os dados pluviométricos relativos ao período do estudo foram obtidos do INMET (Instituto de Meteorologia), medidos na estação meteorológica em atividade mais próxima, que está situada em Belo Horizonte, cerca de 100 km da área de estudo.

\section{Coleta de dados}

Durante o período de junho de 1999 a dezembro de 2002 foram estimadas a riqueza e a abundância das aves aquáticas presentes na Lagoa do Sumidouro e em sua margem. Considerando-se aves aquáticas aquelas que dependem diretamente da água, seja para alimentação e/ou reprodução.

Foram realizadas visitas mensais ao local, em horários fixos, totalizando 40 visitas e aproximadamente 120 horas de observação. Em dias chuvosos não foram feitas observações. As saídas de campo foram feitas sempre no período de 15 às 18 horas devido a maior movimentação de aves aquáticas nesse horário.

O número de espécies (riqueza) e a sua abundância foram registrados através de um censo por contagem direta (Вівву et al. 1993). As espécies foram observadas e contabilizadas com auxílio de binóculos (8 x 25 e 10 x 40) e identificadas com ajuda de guias de campo (HAyman et al. 1986, Sick 1997, SouzA 2002). A nomenclatura científica e a ordenação taxonômica deste trabalho estão de acordo com Sick (1997) e com as modificações sugeridas pelo Comitê Brasileiro de Registros Ornito- 
lógicos (СвRо 2003). Além das 40 visitas padronizadas, foram realizadas outras 17 visitas de quatro horas de duração em diferentes meses e horários do dia com a finalidade de se detectar maior número de espécies.

A riqueza de espécies foi estimada utilizando-se o estimador 'jackknife de primeira-ordem' seguindo o protocolo de Colwell (1997). A diversidade de espécies foi calculada segundo o índice de diversidade de Shannon (MagurRan 1988). A variância do índice de diversidade foi obtida a partir do método "jackknife" utilizando-se o programa EstmateS (Colwell 1997). Este procedimento produz uma estimativa (de riqueza e de diversidade) com um intervalo de confiança de 95\%, permitindo assim comparações estatísticas diretas ao nível de $\mathrm{p}<0,05$.

Também foi determinado o status residencial ou presença sazonal das espécies registradas, podendo ser considerada: (1) Residente (espécie registrada em pelo menos 90\% das visitas, estando presente na região em todas as estações do ano); (2) Visitante (espécie que só foi registrada em determinada época do ano, como por exemplo, numa determinada estação); (3) Provável residente (espécie que, por sua biologia e/ou ecologia poderia ser considerada residente, mas durante o estudo não foi registrada em todas as estações, não apresentando sazonalidade definida); (4) Indeterminada (espécie com presença sazonal não determinada, ou seja, que foram pouco registradas durante o trabalho e, por isso não se pode determinar sua sazonalidade). Os critérios para se definir a sazonalidade das aves foram determinados através de dados de campo e com base nos padrões sazonais definidos por NAKA et al. (2002).

\section{RESULTADOS}

\section{A comunidade de aves aquáticas}

Foram registradas 27 espécies de aves aquáticas, distribuídas em 12 famílias e 7 ordens (Tab. I). A família mais bem representada foi Ardeidae com seis espécies perfazendo 22,2\% do total de espécies. Em seguida, a família Anatidae com cinco espécies $(18,5 \%)$ e Scolopacidae sendo representada por três espécies (11\% do total).

Das 27 espécies registradas, 12 foram consideradas residentes ou prováveis residentes e oito visitantes. Sete espécies não apresentaram nenhum padrão distinto de presença sazonal (Tab. I).

Uma das espécies Platalea ajaja Linnaeus, 1758 é considerada ameaçada de extinção, e outras duas Mycteria americana Linnaeus, 1758 e Ciconia maguari (Gmelin, 1789) são consideradas raras no Estado de Minas Gerais.

A maioria das espécies da comunidade de aves aquáticas foi amostrada nestes três anos de estudo, sendo que a riqueza de espécies estimada pelo 'jackkniffe de primeira ordem' foi de 26,98 \pm 0,98 espécies (Fig. 1).

Não houve diferença significativa entre a riqueza de espécies estimada para as três estações ('chuvosa', 'seca' e 'transição'; Fig. 2). A diversidade de espécies mostrou-se significativa-

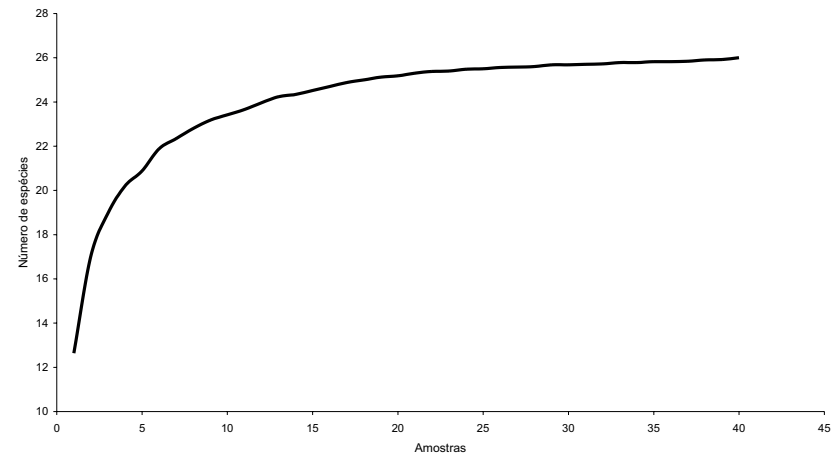

Figura 1. Acúmulo de espécies novas observadas ao longo das amostragens feitas na Lagoa do Sumidouro, APA de Lagoa Santa, Minas Gerais.

mente diferente entre as três estações, sendo a estação de transição com a maior diversidade (Fig. 3).

As aves aquáticas apresentaram, como um todo, uma maior abundância de indivíduos nas estações secas (Fig. 4).

\section{As populações}

As espécies mais abundantes ao longo dos três anos de estudo foram o biguá Phalacrocorax brasilianus (Gmelin, 1789), seguida do mergulhão Podylimbus podiceps (Linnaeus, 1758) e da marreca irerê Dendrocygna viduata (Linnaeus, 1766), sendo a do biguá duas vezes mais numerosa que a das duas seguintes. As populações de biguá e de mergulhão apresentaram picos populacionais na estação seca de 2002 , enquanto a população de irerê atingiu seu pico na estação seca e transitória de 2000 (Fig. 5). A população de biguá mostrou-se muito pequena durante todo o ano de 2001, com no máximo 40-50 indivíduos. No ano de 2002, 548 indivíduos puderam ser observados no mês de julho.

A garça-branca-grande Ardea alba Linnaeus, 1758, a garça-branca-pequena Egretta thula (Molina, 1782), e a galinhad'água Gallinula chloropus (Linnaeus, 1758) apresentaram populações intermediárias e com picos populacionais ao longo das três estações secas seguidas (Figs 6-10).

No presente estudo, a população do colhereiro Platalea ajaja, mostrou-se bastante irregular ao longo do estudo e em relação ao número de indivíduos que permaneciam na lagoa. Os indivíduos apareciam durante a estação de transição (outubro) e permaneciam por toda a estação chuvosa. Entretanto, alguns poucos indivíduos foram registrados durante a estação seca de 2001. O número máximo de indivíduos observados foi 20, nos meses de novembro e dezembro de 2002.

\section{DISCUSSÃO}

Cerca de 30\% das espécies de aves aquáticas que ocorrem na região de cerrado da América do Sul (segundo SiLva 1995) foram registradas na Lagoa do Sumidouro. Este número é bas- 

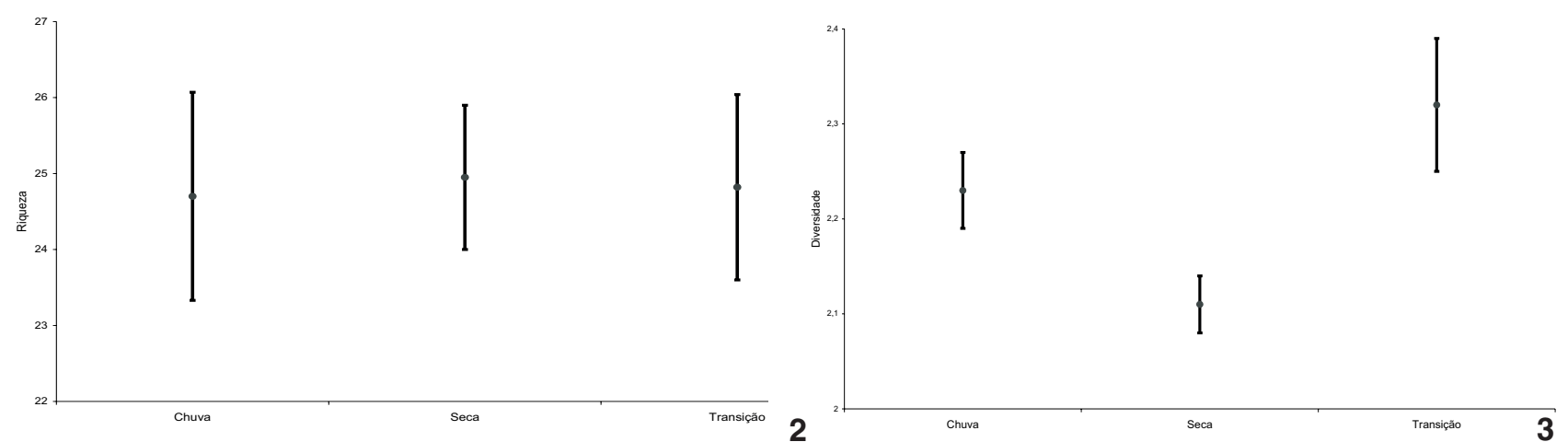

Figuras 2-3. (2) Riqueza estimada de espécies ( \pm desvio padrão) e (3) Diversidade (índice de Shannon-Wiener) estimada ( \pm desvio padrão), para as três estações do ano estabelecidas pela média pluviométrica (veja seção de métodos) na Lagoa do Sumidouro da APA de Lagoa Santa, Minas Gerais.

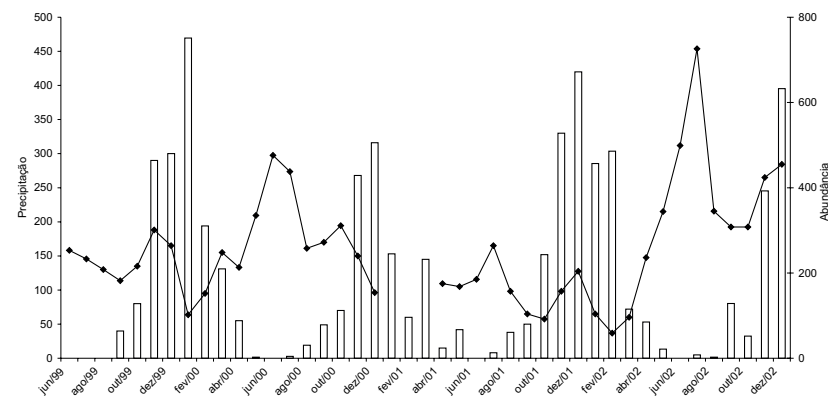

Figura 4. Abundância total de indivíduos (linhas) que freqüentam a Lagoa do Sumidouro na APA de Lagoa Santa, Minas Gerais e a precipitação média mensal (barras) ao longo de junho de 1999 a dezembro de 2002.

tante expressivo e mostra a importância da lagoa para a manutenção destas populações. É previsto que um maior número de espécies possam ser registrados na região se as outras lagoas da APA LS forem amostradas.

O número de espécies aquáticas registradas no presente estudo equivale àquele registrado nos anos de 1996 e 1997 por Hermann et al. (1998) com algumas alterações. No estudo de Hermann et al. (1998), espécies raras como Mycteria americana e Ciconia maguari não foram registradas. Entretanto, tais espécies haviam sido registradas no século XIX por J. Reinhardt (PINTo 1952). Estas espécies são de grande porte e migratórias, e tudo indica que suas populações no Estado de Minas Gerais estão diminuindo, provavelmente devido à perda de habitats (assoreamento e poluição de lagoas) e às mudanças climáticas (e.g. Davies et al. 1998, BirdLife International 2000, PARMesan \& YoHe 2003, Rodrigues et al. 2005).

Outras espécies não registradas por HeRmANn et al. (1998) são os anatídeos migratórios Netta erythrophtalma (WiedNeuwied 1833) e Nomonyx dominica (Linnaeus, 1766), a saracura
Aramides cajanea (Statius Muller, 1766) (Rallidae), e a batuíra Tringa solitaria Wilson, 1813 (Scolopacidae). Todas elas, com exceção da marreca paturi-preta $N$. erythrophtalma, foram registradas por J. Reinhardt (PINTo 1952). A paturi-preta, no entanto, vem apresentando aumento populacional considerável e expansão de sua área de distribuição nos últimos anos (Willis 1991, SICK 1997).

Três espécies amostradas por HeRMANn et al. (1998) não foram registradas no presente estudo, sendo o pato-do-mato Cairina moschata (Linnaeus, 1758), o frango-d'água-azul Porphyrio martinica (Linnaeus, 1766) e a batuíra-de-coleira Charadrius collaris Vieillot, 1818. Todas as três espécies constam na lista de J. Reinhardt (PINTO 1952).

A riqueza e a diversidade de espécies da avifauna aquática presentes na área são bastante importantes em relação à presença de (1) espécies raras e ameaçadas de extinção e (2) espécies migratórias que utilizam a lagoa como local de pernoite e/ ou descanso durante a migração.

Espécies raras no Estado de Minas Gerais ainda podem ser encontradas na Lagoa do Sumidouro, como o colhereiro Platalea ajaja; a cabeça-seca Mycteria americana e o maguari Ciconia maguari. O colhereiro é considerado ameaçado de extinção no Estado de Minas Gerais (Machado et al. 1998). Quatorze indivíduos desta espécie foram registrados por Hermann et al. (1998). Esses dados sugerem que esta pequena população que ocorre na Lagoa do Sumidouro pode ser residente. Isso mostra a importância da Lagoa como área de forrageamento desta pequena população. Entretanto, apenas quatro indivíduos de cabeça-seca foram registrados em novembro de 2000, três em outubro de 2001 e dois em novembro de 2002. Quanto ao maguari, três indivíduos foram observados uma única vez em novembro de 2000. Sabe-se que estas espécies apresentam padrões migratórios característicos na Amazônia e no Pantanal sul do Brasil (ANTAs 1994, Sick 1997). A ocorrência destas espécies em lagoas naturais ou artificiais no sudeste do Brasil ainda é pouco documentada, mas o maguari foi registra- 

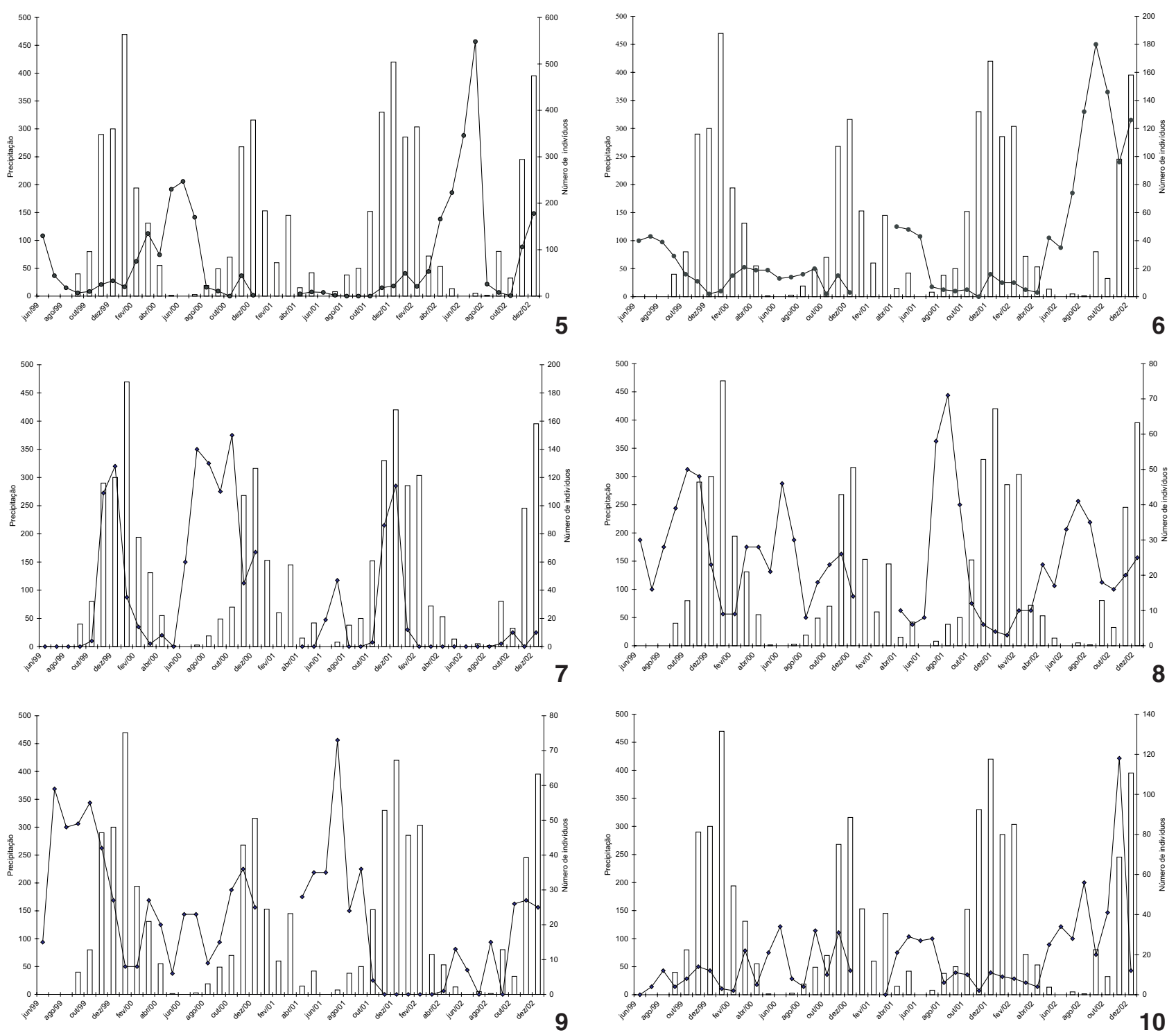

Figuras 5-10. Número de indivíduos (linhas) e a precipitação média mensal (barras) ao longo de junho de 1999 a dezembro de 2002 na Lagoa do Sumidouro na APA de Lagoa Santa, Minas Gerais: (5) biguá, Phalacrocorax brasilianus; (6) mergulhão, Podylimbus podiceps; (7) marreca irerê Dendrocygna viduata; (8) garça-branca-grande, Ardea alba; (9) garça-branca-pequena Egretta thula; (10) galinha-d’água Gallinula chloropus.

da no Parque Nacional da Serra do Cipó, a cerca de $60 \mathrm{~km}$ ao norte da APA LS em 1999 (RodRIGuEs et al. 2005). A ocorrência da cabeça-seca e do maguari na mesma época do ano (outubro e novembro) sugere a existência de um padrão sazonal. Estudos mais detalhados de monitoramento em outras lagoas da região poderão corroborar essa hipótese.

A Lagoa do Sumidouro abriga oito espécies migratórias, que ocupam suas margens principalmente nos meses de junho a setembro e fazem deste habitat um local de pernoite ou para- da durante a jornada migratória. Algumas delas permanecem por poucos dias, como Mycteria americana e Tringa solitaria. Outras espécies ocupam a lagoa durante um tempo maior, como Tringa flavipes (Gmelin, 1789) e Himantopus himantopus (Linnaeus, 1758). Esta última espécie, embora tenha sido considerada residente, tem sua população aumentada drasticamente nos meses da época seca. Há também o caso do biguá Phalacrocorax brasilianus que ocorreu de maneira irregular durante os três anos de estudo. $P$. brasilianus é uma das espécies 
Tabela I. Espécies de aves aquáticas registradas na Lagoa do Sumidouro, APA de Lagoa Santa, Minas Gerais e a indicação da presença sazonal. A ordenação taxonômica segue Sick (1997) е Свво (2003). Presença Sazonal: (Res) Residente, (Vis) visitante, (Pr) provável residente, (Ind) indeterminada.

\begin{tabular}{|c|c|c|}
\hline Família/Espécie & Nome popular & Presença sazonal \\
\hline \multicolumn{3}{|l|}{ Podicipedidae } \\
\hline Podylimbus podiceps (Linnaeus, 1758) & mergulhão-caçador & Res \\
\hline \multicolumn{3}{|l|}{ Phalacrocoracidae } \\
\hline Phalacrocorax brasilianus (Gmelin, 1789) & biguá & Ind \\
\hline \multicolumn{3}{|l|}{ Ardeidae } \\
\hline Ardea cocoi Linnaeus, 1766 & garça-moura & Vis \\
\hline Ardea alba Linnaeus, 1758 & garça-branca-grande & Res \\
\hline Egretta thula (Molina, 1782) & garça-branca-pequena & $\operatorname{Pr}$ \\
\hline Butorides striatus (Linnaeus, 1758) & socozinho & $\operatorname{Pr}$ \\
\hline Syrigma sibilatrix (Temminck, 1824) & maria-faceira & $\operatorname{Pr}$ \\
\hline Nycticorax nycticorax (Linnaeus, 1758) & savacu & Vis \\
\hline \multicolumn{3}{|l|}{ Threskiornithidae } \\
\hline Phimosus infuscatus (Lichtenstein, 1823) & tapicuru-de-cara-pelada & Ind \\
\hline Platalea ajaja Linnaeus 1758 & colhereiro & Vis \\
\hline \multicolumn{3}{|l|}{ Ciconiidae } \\
\hline Mycteria americana Linnaeus, 1758 & cabeça-seca & Vis \\
\hline Ciconia maguari (Gmelin, 1789) & maguari & Ind \\
\hline \multicolumn{3}{|l|}{ Anatidae } \\
\hline Dendrocygna viduata (Linnaeus, 1766) & irerê & $\operatorname{Pr}$ \\
\hline DENDROCYGNA AUTUMNALIS (Linnaeus, 1758) & asa-branca & Vis \\
\hline Netta erythrophthalma (Wied, 1832) & paturi-preta & Ind \\
\hline Amazonetta brasiliensis (Gmelin, 1789) & pé-vermelho & $\operatorname{Pr}$ \\
\hline Nomonyx dominica (Linnaeus, 1766) & marreca-de-bico-roxo & Vis \\
\hline \multicolumn{3}{|l|}{ Aramidae } \\
\hline Aramus guarauna (Linnaeus, 1766) & carão & Ind \\
\hline \multicolumn{3}{|l|}{ Rallidae } \\
\hline Aramides cajanea (Statius Muller, 1766) & saracura & Ind \\
\hline Gallinula chloropus (Linnaeus, 1758) & frango-d'água-comum & Res \\
\hline \multicolumn{3}{|l|}{ Jacanidae } \\
\hline Jacana jacana (Linnaeus, 1766) & jaçanã & Res \\
\hline \multicolumn{3}{|l|}{ Scolopacidae } \\
\hline Tringa solitaria Wilson, 1813 & maçarico-solitário & Vis \\
\hline Tringa flavipes (Gmelin, 1789) & maçarico-de-perna-amarela & Vis \\
\hline Gallinago paraguaiae (Vieillot, 1816) & narceja & Ind \\
\hline \multicolumn{3}{|l|}{ Recurvirostridae } \\
\hline Himantopus himantopus (Linnaeus, 1758) & pernilongo & $\operatorname{Pr}$ \\
\hline \multicolumn{3}{|l|}{ Alcedinidae } \\
\hline Ceryle torquata (Linnaeus, 1766) & martim-pescador-grande & $\operatorname{Pr}$ \\
\hline Chloroceryle amazona (Latham, 1790) & martim-pescador-verde & $\mathrm{Pr}$ \\
\hline
\end{tabular}

mais comum de aves aquáticas, mas sua biologia no Brasil é praticamente desconhecida (Olmos \& Silva-e-Silva 2003). A espécie é migratória em alguns locais, como em manguezais do
Estado de São Paulo (Olmos \& Silva-e-Silva 2001) e residente em outros, como na Lagoa Rodrigo de Freitas no Rio de Janeiro (Alves \& Pereira 1998). 
A manutenção destas espécies de aves aquáticas pode estar comprometida devido ao alto índice de degradação ambiental promovido por interferências antrópicas de diversas naturezas na região, sendo que a expansão demográfica e a atividade mineradora são os principais exemplos (SouzA 1998). Nos últimos 10 anos, o número de pessoas que visita a lagoa para a prática da pesca e recreação vem aumentando drasticamente, bem como a construção de casas ao seu redor (Hermann et al. 1998). Isso tem resultado em sobrepesca, assoreamento devido a construções irregulares ao redor da lagoa, e aumento da carga de esgoto urbano despejado.

A presença de uma espécie ameaçadas de extinção e local de parada de espécies migratórias faz com que a região da APA Carste de Lagoa Santa seja considerada uma área de extrema importância para a conservação destas espécies.

\section{AGRADECIMENTOS}

Somos gratos ao Conselho Nacional para o Desenvolvimento Científico e Tecnológico (CNPq), pela concessão de uma bolsa de produtividade em pesquisa a M. Rodrigues (1998-2001). Agradecemos especialmente aos consultores J.M.C. da Silva. E. Hofling e M. Marini, e a J.E. Cortes-Figueira pela leitura e sugestões ao manuscrito, ao Departamento de Zoologia da UFMG pelo apoio e infra-estrutura, e a F. Michelin pelo apoio no trabalho de campo.

\section{REFERÊNCIAS BIBLIOGRÁFICAS}

Alves, M.A.S. \& E.F. Pereira. 1998. Richness, abundance and seasonality of bird species in a lagoon of an urban area (Lagoa Rodrigo de Freitas) of Rio de Janeiro, Brazil. Ararajuba, Brasília, 6 (2): 110-116.

AnTAS, P.T.Z. 1994. Migration and other movements among the lower Paraná River valley wetlands, Argentina, and the south Brazil/Pantanal wetlands. Bird Conservation International, Cambridge, 4: 181-190.

Bibby, C.J.; N.D. Burgess \& D.A. Hill. 1993. Bird census techniques. London, Academic Press, 257p.

Birdife International. 2000. Threatened birds of the world. Cambridge, Lynx Editions, BirdLife International, 852p.

CвRо. 2003. Lista de aves do Brasil. Comitê Brasileiro de Registros Ornitológicos. Disponível em: http://www.ib.usp.br/ cbro. Acesso em 20 de fevereiro de 2004.

Colwell, R.K. 1997. EstimateS: statistical estimation of species richness and shared species from samples. Version 5. User's Guide and application. Available on line at: http:// viceroy.eeb.uconn.edu/estimates.

Cartelle, C. 1994. Tempo passado: mamíferos do Pleistoceno em Minas Gerais. Belo Horizonte, Acesita, 121p.

Davies, A.J.; L.S. Jenkinson; J. H. Lawton; B. Shorrocks \& S. Wood. 1998. Making mistakes when predicting shifts in species range in response to global warming. Nature, London, 391: 783-786.
Fernandez, F.A.S. 1997. Efeitos da fragmentação de ecossistemas: a situação das Unidades de Conservação. Anais do Congresso Brasileiro de Unidades de Conservação, Curitiba, Unilivre, p. 48-68.

Furness, R.W.; J.J.D. Greenwood \& P.J. Jarvis 1993. Can birds be used to monitor the environment?, p. 1-41. In: R.W. FurNess \& J.J.D GREENWOOD (Eds). Birds as monitors of environmental change. London, Chapman \& Hall, 325p.

Goldsmith, F.B. 1991. Monitoring for conservation and ecology. London, Chapman \& Hall, 276p.

Hayman, P.; J. Marchant \& T. Prater. 1986. Shorebirds: an identification guide. Boston, Houghton Mifflin, 412p.

Herrmann, G.; H.C. Kohler. \& J.C. Duarte. 1998. Estudo do meio biótico da APA Carste de Lagoa Santa. Belo Horizonte, Instituto Brasileiro do Meio Ambiente e dos Recursos Naturais Renováveis, Companhia de Pesquisa de Recursos Minerais, 92p.

Machado, A.B.M.; G.A.B. Fonseca; R.B. Machado; L.M.S. Aguiar \& L.V. Lins. 1998. Livro vermelho das espécies ameaçadas de extinção da fauna de Minas Gerais. Belo Horizonte, Fundação Biodiversitas, 605p.

Madeira, A.J. \& W.G. Fernandes. 1999. Reprodutive phenology of sympatric taxa of Chamaecrista (Leguminosae) in Serra do Cipó, Brasil. Jornal of Tropical Ecology, Cambridge 15 (2): 463-479.

Magurran, A.E. 1988. Ecological diversity and its measurement. Princeton, Princeton University Press, 121p.

NaKA, L.N.; M. Rodrigues; A.L. Roos \& M. A. Azevedo. 2002. Bird conservation on the island of Santa Catarina. Bird Conservation International, Cambridge, 12 (1): 123-150.

Neves, W.A.; J.F. Powell; A. Prous \& E.G. Ozolins. 1998. Lapa Vermelha IV, Hominid 1: Morphological affnities of the earliest known American. American Journal of Physical Anthropology, Washington, 26 (Suppl.): 169.

Olmos. F. \& R. Silva-E-Silva. 2001. The avifauna of a southeastern Brazilian mangrove swamp. International Journal of Ornithology, Belo Horizonte, 4 (3/4): 137-207.

Olmos. F. \& R. SilvA-E-SILVA. 2003. Guará: ambiente, flora e fauna dos manguezais de Santos-Cubatão, Brasil. São Paulo, Empresa das Artes, 216p.

Parmesan, C. \& G. Yohe. 2003. A globally coherent fingerprint of climate change impacts across natural systems. Nature, London, 421: 37-42.

PiLó, L.B. 1997. Rochas carbonáticas e relevos cársticos em Minas Gerais. O Carste, Belo Horizonte, 9 (3): 72-78.

Pinto, O.M.O. 1952. Súmula histórica e sistemática da ornitologia de Minas Gerais. Arquivos de Zoologia, São Paulo, 8 (1): $1-51$.

Pough, F.H.; J.B. Heiser \& W.N. Mcfarland. 1999. A Vida dos Vertebrados. São Paulo, Atheneu, 798p.

Rodrigues, M.; L.A. Carrara; L.P. Faria \& H.B. Gomes. 2005. Aves do Parque Nacional da Serra do Cipó: o Vale do Rio Cipó, Minas Gerais, Brasil. Revista Brasileira de Zoologia, Curitiba, 22 (1): 326-333. 
RuTSCHKE, E. 1987. Waterfowl as bio-indicators. International Council for Bird Preservation Technical Publication no. 6, Cambridge, International Council for Bird Preservation, p.167-172.

SICK, H. 1997. Ornitologia brasileira. Rio de Janeiro, Nova fronteira, 912p.

Silva, J.M.C. 1995. Birds of the Cerrado Region, South America. Steenstrupia, Copenhagen, 21 (2): 69-92.

Soulé, M.E. 1987. Viable populations for conservation. Cambridge, Cambridge University Press, 311p.

SouzA, H.A. 1998. Zoneamento Ambiental da APA Carste de Lagoa Santa-MG. Belo Horizonte, Instituto Brasileiro do Meio Ambiente e dos Recursos Naturais Renováveis, Com- panhia de Pesquisa de Recursos Minerais, 62p.

SouZA, D. 2002. All the birds of Brazil: an identification guide. Feira de Santana, Dall, 356p.

Stotz, D.F.; J.W. FitzPatrick; T.A. PARKer III \& D.K. Moskovits. 1996. Neotropical Birds: ecology and conservation. Chicago, The University of Chicago Press, 478p.

WARMING, E. 1908. Lagoa Santa, contribuição para a geographia phytobiológica. Belo Horizonte, Imprensa Oficial do Estado de Minas Gerais, II+282p.

Willis, E.O. 1991. Expansão geográfica de Netta erythrophtalma, Fluvicola nengeta e outras aves de zonas abertas com a 'desertificação' antrópica em São Paulo. Ararajuba, Rio de Janeiro, 2 (1): 101-102.

Recebido em 21.II.2005; aceito em 10.X.2005. 